\title{
A Novel Approach for Spectrum Sensing with Effect of Radiation
}

\author{
R. Prakash Kumar ${ }^{1}$ and G. Santhosh Kumar $^{2}$ \\ ${ }^{1,2}$ CVR College of Engineering / ECE Department, Hyderabad, India. \\ ${ }^{1}$ Email: prakash.rachmagdu@gmail.com \\ ${ }^{2}$ Email: santhosh_gangi@yahoo.com
}

\begin{abstract}
In Orthogonal Frequency Division Multiplexing (OFDM) large serial data can be converted into parallel data stream. Each parallel block can be transmitted with orthogonal carriers. If the spectrum is not utilized effectively then more number of carriers can be used in wireless applications, but increasing the carriers leads to radiation effect in environment. To reduce the radiation effect, spectrum should be effectively utilized. Effective utilization of electromagnetic spectrum for the unlicensed users to use the licensed spectrum, white spaces i.e. unused frequency bands need to be detected by dynamic spectrum access in Cognitive Radio(CR) .In this paper two spectrum sensing techniques are proposed, they are energy detection with Wavelet transform and Cyclostationarity sensing.
\end{abstract}

Index Terms-: Spectrum Sensing, radiation, CR and OFDM.

\section{INTRODUCTION}

Day by day wireless devices and applications are increased, but the availability of Electro Magnetic spectrum is a limited natural resource. As per Federal Communications Commission (FCC) spectrum access and radiation effect are more significant problem than physical scarcity of spectrum [5]. Radiation effect can be measured by Specific Absorption Rate (SAR) in mobile communication. Subscribers can be seen the SAR value in the standard mobile station (mobile hand set) by *\#07\# command. While designing mobile handsets, manufactures are always trying to keep the SAR value less than 1.6 Watt/Kg in European countries to avoid radiation[9][11]. This value is different for different countries. To measure SAR value, mobile is tested with some conditions where the mobile is kept near to the user in the presence of base station. The actual SAR values are usually well below those stated below. The maximum SAR for this model as per the compliance standard and the conditions which it was recorded were-Head $\mathrm{SAR}=0.376 \mathrm{~W} / \mathrm{Kg}$. In personal communication, CR is built on Software Defined Radio (SDR) which is a robust enhance technology for multi service, reconfigurable and, reprogrammable software. The goal of CR network or wireless node changes its transmission or reception parameters to communicate efficiently anywhere, anytime for avoiding interference with licensed or unlicensed users with efficient utilization of the radio spectrum[7][8]. It uses the methodology of sensing and learning from the environment and adapting to statistical variations in real time. $\mathrm{CR}$ is like feedback communication systems which is used to provide forward and backward link between transmitter and receiver .CR
Concept was first developed by Defense Advance Research Products Agency (DARPA) scientist, Dr. Joseph Mitola which leads to concept of IEEE 802.22, which is used for wireless Regional Area Network (WRAN) for white space in Television Frequency Spectrum with no interference. In case TV applications of $700 \mathrm{MHz}$ [3] band IEEE 802.22.1 is not effective because interference is harmful. To reduce this interference IEEE802.22.2 is recommended [4].

In wireless communication system an efficient use of frequency spectrum can be achieved by radio network which is called Cognitive Radio network. It is an intelligent multi user communication system with following abilities.

- Each user receiver continuously sense the surrounding environment.

- Observe the environment and adapt to it in response.

- Multiple users can Communicate can through a selforganizing manner.

The characteristics of $\mathrm{CR}$

$>$ Flexibility

$>$ Reconfigurability

$>$ Awareness

$>$ Adaptability

$>$ Intelligence

In order to achieve these characteristics CR is modify and access the radio spectrum without causing excessive interference to the primary users and allocates spectrum to the secondary users. The allocation of spectrum of cognitive radio cycle is shown in Fig.1. This includes Sensing of spectrum, Analysis of spectrum and Decision making spectrum. Sensing of spectrum is to detect the unused spectrum holes (white holes). Based on these holes, it is possible for channels to target without collision. Spectrum can be shared by two methods such as cooperative and non-cooperative [1] [3].

Spectrum can be analyzed through the spectrum holes and it allocates the required band to user. While analysis the spectrum holes the following parameters are analyzed. Those are Path loss, Interference, Wireless link errors and Link layer delay.

Spectrum decision depends on Quality of Service (QoS) for secondary users with the parameters Data rate, Acceptable error rate and Delay time etc. 


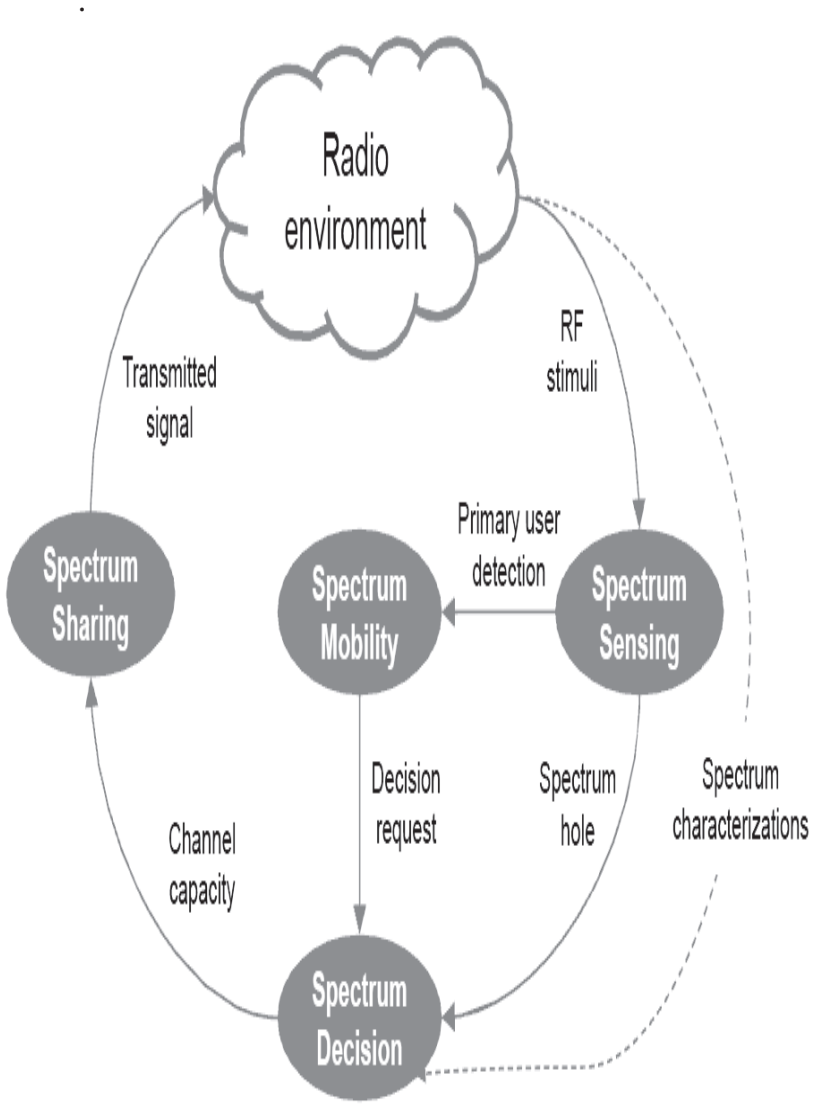

Figure 1.Cognitive radio Cycle.

In this paper various methods of spectrum sensing are shown in Fig 2. We start by introducing the methods of spectrum sensing methods for CR network in section II, Energy detection based Wavelet Packet transform sensing in section III, Section IV Cyclostationarity sensing and Section $\mathrm{V}$ simulation results and finally our conclusions are presented.

\section{SENSING OF SPECTRUM}

In this paper, the main focus is sensing of spectrum in CR network. Spectrum sensing can be obtained by using of existence of primary users on a geographical area and database, and by using beacons or local spectrum sensing at CR. Sensing methods not only measures the spectral content, energy over radio frequency spectrum [5][7] but also determines signals occupied in the frequency spectrum including the modulation and carrier frequency. Basically sensing are two methods like cooperative and non cooperative or signal processing techniques shown in Fig2. For CR network, sensing of spectrum requires large dynamic range with high resolution Analog to Digital Converters (ADCs), very high sampling rate and high speed signal processers. While sensing the spectrum the following parameters are to be considered [10].

- Detecting Spread Spectrum Primary Users

- Sensing Duration and Frequency

- Detecting Spread Spectrum Primary Users

\section{A. Principle of sensing of spectrum}

Fig. 3 shows the principle of the sensing of spectrum. The figure shows that to protect PU transmission, CR Transmitter is required to perform spectrum sensing to check whether there is any active PU receiver in the coverage of CR Transmitter. If there is any primary user transmission in the coverage of CR Transmitter, then CR Transmitter cannot transmit at that time because it will cause interference to the primary user transmission.

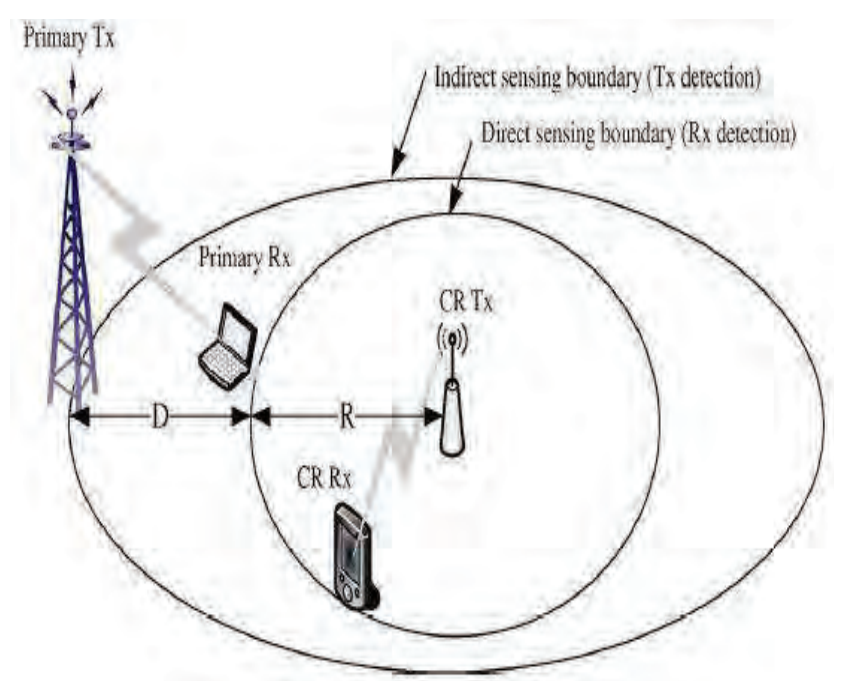

Figure 2. Principle of sensing of spectrum

\section{B.. various methods of sensing spectrum}

The main goal of CR network is to detect the spectrum holes without causing interference to the Primary Users (PU) and assign to the Secondary Users (SU). Spectrum sensing can be broadly classified into three major types as shown in Fig.2, non cooperative or transmitter detection, cooperative sensing and interference based sensing[10][11].

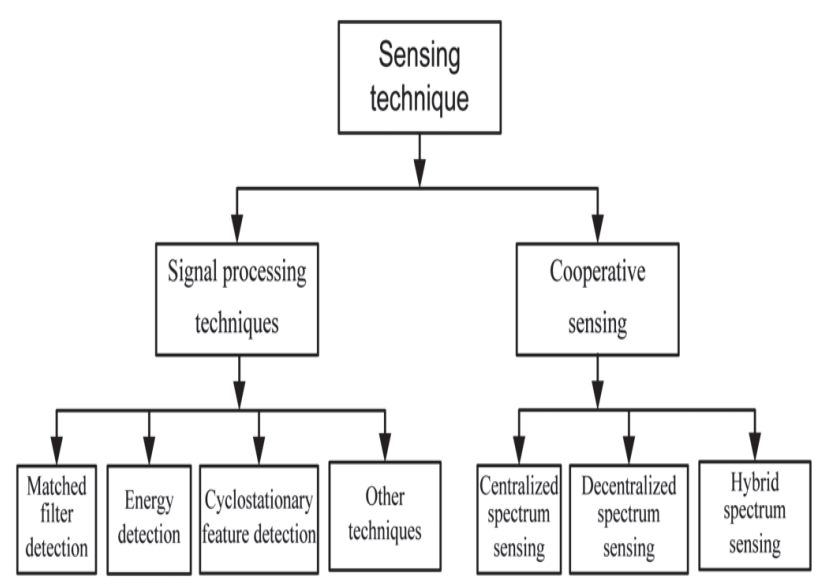

Figure 3. Various methods of sensing spectrum.

Sensing of spectrum plays major role in the cognitive cycle as shown in Fig 1. To improve the sensing of spectrum performance in CR two methods are used, one 
cooperative and non cooperative, In Cooperative CR sensors can sense the multiple channels simultaneously and share their sensing information to SU. Cooperative sensing is of three types as shown in Fig. 2. centralized, decentralized and hybrid [7].

In centralized sensing of spectrum each CR sensor senses the spectrum locally and independently making a decision whether the PU's signal is present or absent on a particular channel. This information is passed through the centrally located server or cluster head and takes the decision to infer the absence or presence of the PU. Whenever a CR wireless sensor wants to send data, it requests channel information to the central cooperator [9].

In decentralized cooperative sensing the information can be shared by intra-cluster to other clusters and makes the decision based on demand. In this there is no central control independently take the decision. But it requires a periodic update on the spectrum information table, hence requires more storage and computation [6].

In hybrid cooperation, $\mathrm{CR}$ wireless sensors share the information among all sensors in the network. The challenge of CR wireless sensor is computation complexity and allocating resources [8].

In this paper, each radio senses itself about the channel information to know the channel status like idle or busy in noncooperative sensing spectrum method. In cooperative method, CR sensing data with others and use the outcome sensing of others. The detection of PU is depended on the primary transmitter detection. In this approach filter matching is necessary. Due to the low computation complexity and implementation, the energy detection method is most fabulous method. In this approach receiver does not require any knowledge on the PU data. Based on the signal and noise floor comparison, the original signal detected by threshold detector, where choosing the threshold value is key role in this method. The system performance mainly depends on signal to noise ratio value, if the value of SNR is low then, It tries to increase the value of SNR by varying system parameters. While measuring the threshold value two parameters are needed [12][13]. They are Pd (probability correct detection) and Pf (probability false detection) these play critical role in sensing of spectrum.

\section{WAVELET PACKET TRANSFORM}

Wavelet transform has become more popular in signal processing because it gives signal information in time-frequency. In communication, noise component is having high frequency and signal component is having low frequency. The wavelet transform decomposes a signal into approximation representation that shows signal details and trends as a function of time. Thresholding is the simple method to remove the noise and reconstruct the signal is reducing the size of coefficient detail. These coefficients cannot be zero because it contains original signal information. The two popular methods to reduce the noise are hard and soft thresholding. The principle in Wavelet Packet Transform (WPT) is approximate both high and low pass sub-bands at all scales in the filter bank approximation and implementation. Hence this method is suitable for non-stationary time variable signal to identify the signal information in both high and low frequency bands. The decomposition tree is shown in Fig 4. Signal S to be combination of as A1 + AAD3 + DAD3 + DD2.This combination of representation is not possible with general wavelet but feasible made with WPT [5].

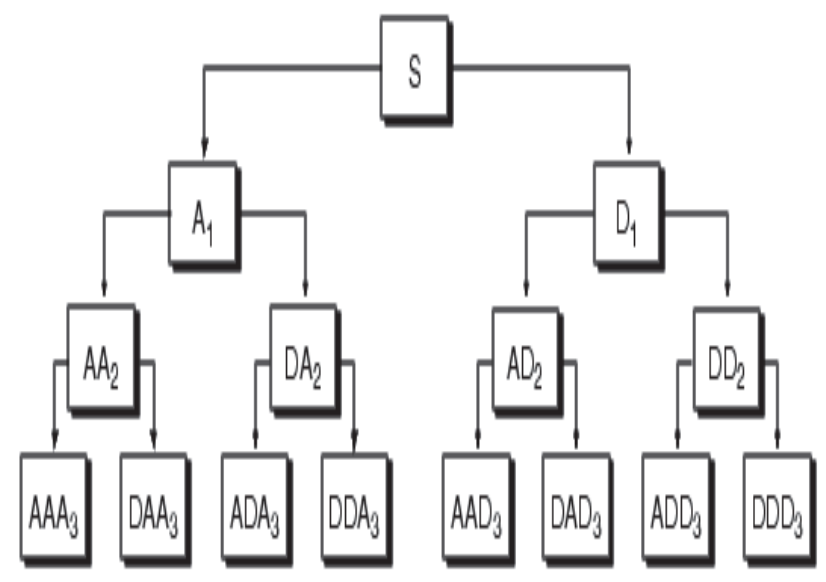

Figure. 4 Wavelet packet decomposition tree

\section{C) Energy Detection Model based on WPT}

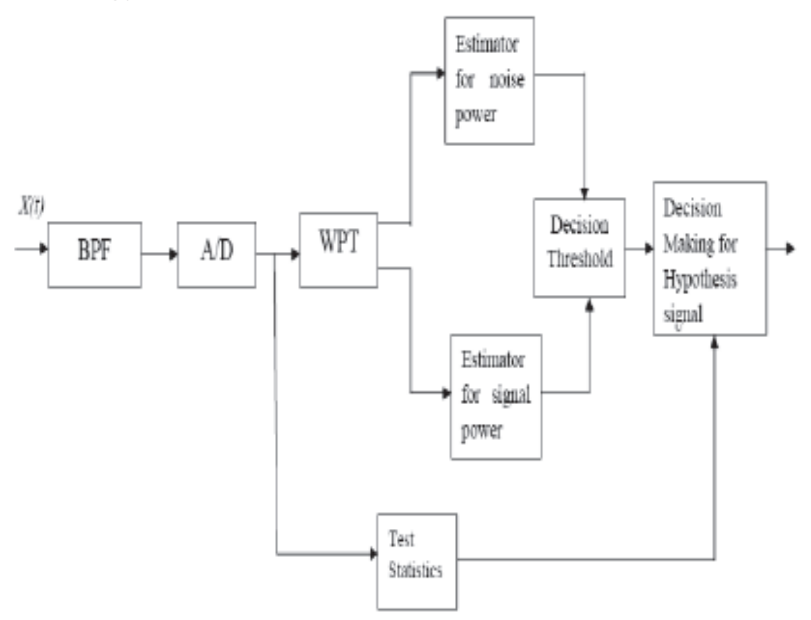

Figure 5 .Block Diagram of energy detection based on WPT

Energy detection based on WPT block diagram is shown in fig 5. Band Pass Filter (BPF) removes the unwanted frequencies and selects the frequencies of bandwidth $\mathrm{W} \mathrm{Hz}$ then passed through Analog to Digital Converter (A/D) coverts into digital signal consists of both signal and noise $\mathrm{x}(\mathrm{n})$.

$x(n)=\xi(n)+w(n) \quad n=0,1 \ldots N-1 \quad---(1)$

Where $\mathrm{s}(\mathrm{n})$ is the PU signal with zero mean and variance $\sigma_{\mathrm{s}}^{2}$

$\mathrm{W}(\mathrm{n})$ is AWGN with zero mean and variance $\sigma_{\mathrm{w}}{ }^{2}$

If Spectrum can be used by $\mathrm{PU}$ then $\mathrm{s}(\mathrm{n}) \neq 0$ otherwise $s(n)=0$. Digital signal $x(n)$ can be processed as follows first $\mathrm{x}(\mathrm{n})$ is sent to Wavelet Packet transform 
(WPT) to estimate current noise power $\left(\sigma_{\mathrm{w}}{ }^{2}\right)$ and signal power $\left(\sigma_{\mathrm{s}}^{2}\right)$ calculate the energy of $\mathrm{x}(\mathrm{n})$ is decomposed for a certain level related to the resolution required and then is reconstructed by wavelet packet decomposition coefficients. And hence the noise power and reconstructed signal power is estimated [9][10]. If $\mathrm{X}>\gamma^{*}$, we can make a decision that the channel is occupied by one PU or more. Otherwise, the channel is vacant, and SUs could make use of the channel at this moment.

\section{IV.CYCLOSTATIONARITY SPECTRUM SENSING}

In energy based detection first decision about the signal contains noise and estimates it. Where as in case of sensing of spectrum using Cyclostationarity is unused or hidden frequencies (called cyclic frequencies) exits in modulation, shifting in frequency, spreading codes and pulse shaping in communication systems. These frequencies can be detected by using mathematical tool such as cyclic autocorrelation and the spectral correlation function. But computational complexity is more in the Cyclostationarity analysis operations due to the nature of the estimation as shown below fig 6 .

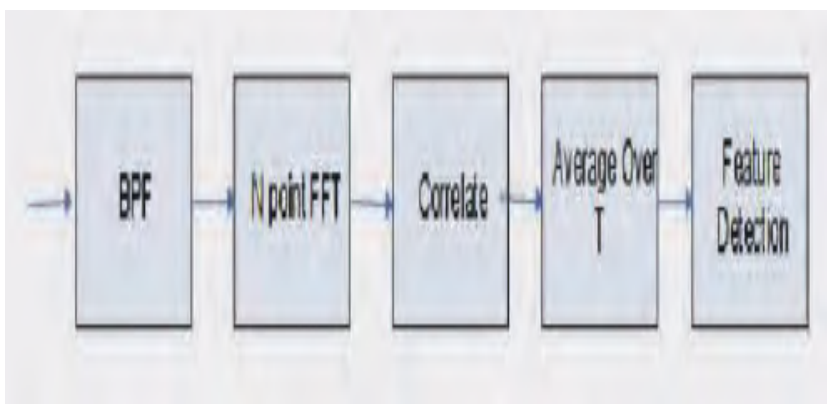

Figure 6. Cyclostationarity Feature Detector

\section{Simulation Results}

In this paper all the simulations are done in MatLab. First simulate $P_{d}$ verses different values of SNR using WPT for different samples as shown in Fig 7.and for the same use WPT under different wavelets as shown in Fig8. Finally simulation is done by Cyclostationarity sensing method with QPSK modulation as shown in Fig 9.

In WPT based energy detection the spectrum can be sensed by different sample values had simulated as shown in fig 7. As sample number $\mathrm{N}$ is large then $\mathrm{P}_{\mathrm{d}}$ is close to 1 .so that better sensing has to be done for only large values of $\mathrm{N}$. But as $\mathrm{N}$ increases SAR value increases. The simulation algorithm steps are as follows.

- random signal is generated and add the noise

- The signal is added with AWGN signal is obtained whose WPT is finding out.

- Variance is calculated based on level of threshold.

- Plot the graph between Pd vs. SNR using WPT.

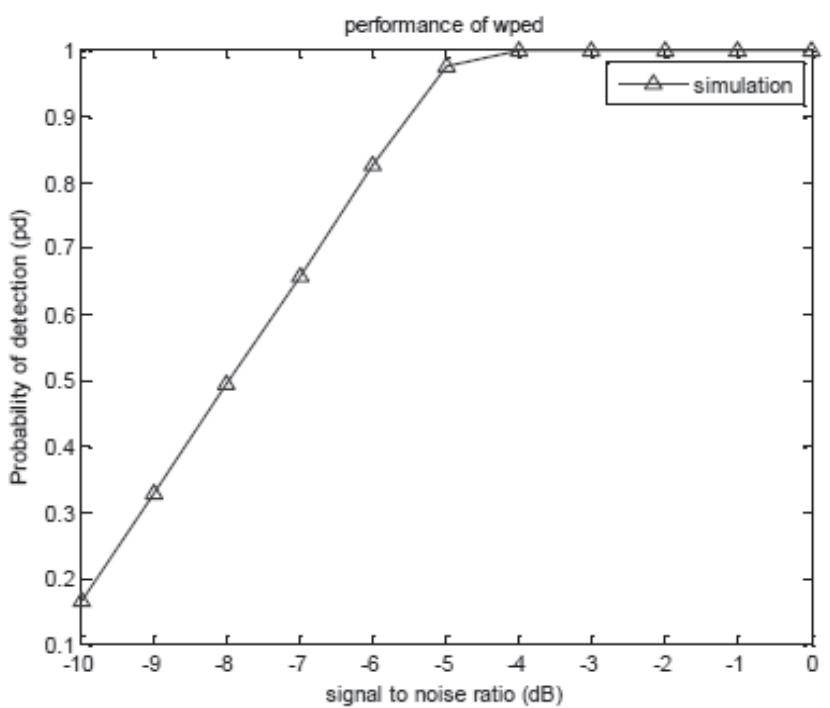

Figure 7. Simulation of PD vs SNR using WPT under different sample numbers.

Same signal has been simulated for different wavelets used for energy detection based sensing is shown in Fig 8 .In this paper the simulation results shows the $\mathrm{db} 2$ wavelet. It gives less error for decomposition and reconstruction of the signal.

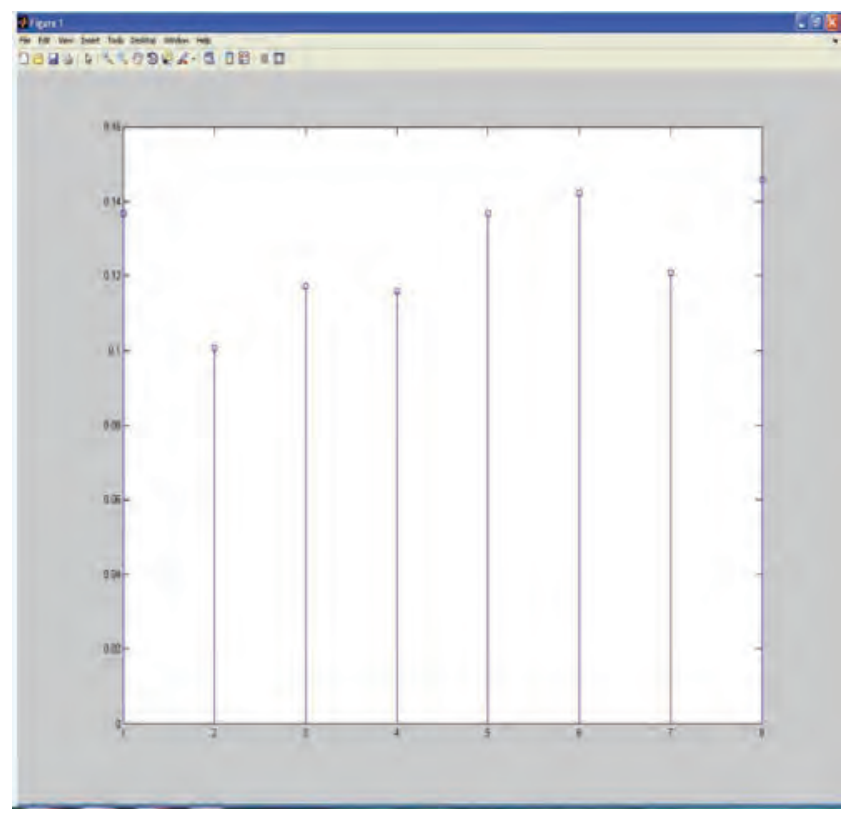

Figure 8. Simulation of PD vs SNR using WPT under different wavelets.

\section{Simulation of Cyclostationarity sensing}

In energy based sensing is based on $\mathrm{P}_{\mathrm{d}}$ and $\mathrm{Pf}$ value under signal is noisy environment. Where as in Cyclostationarity the signal is transmitted with Quadrature Phase Shift Keying (QPSK) modulated with a carrier frequency of $200 \mathrm{~Hz}$, we get two peaks at $400 \mathrm{~Hz}$. These peaks signifies that the PU uses the QPSK and the reason for getting peaks at double the carrier frequency is autocorrelation of the received signal. 


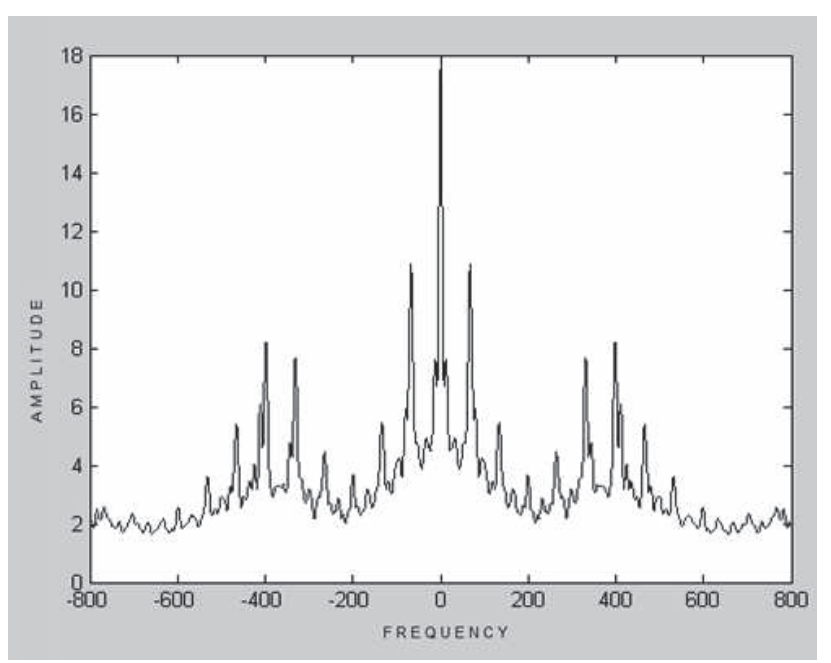

Figure 9. Simulation of Cyclostationarity spectrum sensing with QPSK modulation

\section{CONCLUSIONS}

WPT based energy spectrum sensing is best method in noisy environment compared to the conventional methods. In this paper the probability is closely reached to the value 1 as the number samples are increased at low SNR Cyclostationarity sensing is good method compared with the regular method. As we utilize the spectrum efficiently then automatically number of carriers are reduced which results in reduced radiation effect.

\section{REFERENCES}

[1]. Simon Haykin, "Cognitive Radio: Brain-Empowered Wireless Communications", IEEEJournal on Selected Areas in Communications.vol. 23, no. 2, February 2005,pp. 201-220.

[2]. Hisham A. Mahmoud, Tevfik Y"ucek, and H"useyin Arslan "OFDM for Cognitive Radio: Merits and Challenges" Department of Electrical Engineering, University of SouthFlorida.

[3]. A. Rajan and C. Tepedelenlioglu, "Diversity combining over Rayleigh fading channels with symmetric alpha-stable noise," IEEE Transactions on Wireless Communications, vol. 9, no. 9, pp. 2968-2976, 2010.

[4]. Zhang Shi-bing and Qin Jin-jing, "Energy Detection Algorithm Based on Wavelet Packet Transform under Uncertain Noise for Spectrum Sensing", IEEE Conference of Wi3COM2010.

[5]. M. Sherman, A. N. Mody, R. Martinez, and C. Rodriquez, "IEEE standards supporting cognitive radio and networks, dynamic spectrum access, and coexistence," IEEE Communication Magazine., Vol. 46, No. 7, pp. 72-79, July 2008.

[6]. Bhargavi, D., \& Murthy, C. R. (2010, June). Performance comparison of energy, matched-filter and cyclostationaritybased spectrum sensing. In Signal Processing Advances in Wireless Communications (SPAWC), 2010 IEEE Eleventh International Workshop on (pp. 1-5). IEEE.

[7]. Digham, F. F., Alouini, M. S., \& Simon, M. K. (2007). On the energy detection of unknown signals over fading channels. Communications, IEEE Transactions on, 55(1), 21-24.

[8]. Digham, F. F., Alouini, M. S., \& Simon, M. K. (2007). On the energy detection of unknown signals over fading channels. Communications, IEEE Transactions on, 55(1), 21-24.

[9]. Yucek, T., \& Arslan, H. (2009). A survey of spectrum sensing algorithms for cognitive radio applications. Communications Surveys \& Tutorials, IEEE, 11(1), 116130.

[10].Kapoor, S., \& Singh, G. (2011, February). Non-cooperative spectrum sensing: A hybrid model approach. In Devices and Communications (ICDeCom), 2011 International Conference on (pp. 1-5). IEEE.

[11].K.-L. A. Yau, N. Ramli, W. Hashim, and H. Mohamad, "Clustering algorithms for cognitive radio networks: A survey," J. Netw. Comput. Appl., vol. 45, pp. 79-95, Oct. 2014.

[12].G. Tsiropoulos, O. Dobre, M. Ahmed, and K. Baddour, "Radio resource allocation techniques for efficient spectrum access in cognitive radio networks," IEEE Commun. Surveys Tuts., vol. 18, no. 1, pp. 817-840, 1st Quart. 2016.

[13].S. Z. A. Jalil, M. Y. M. A. Karim, H. Abdullah, and M. N. Taib, "Instrument System Setup for Human Radiation Wave Measurement," in Proceedings of the IEEE SCOReD Student Conference, 2009, pp. 523-525. 